\title{
A GEL OF GLYCOSAMINOGLYCANS LINING THE ANTERIOR AND POSTERIOR CHAMBERS IN MAN: HISTOCHEMICAL EVIDENCE AT LIGHT AND ELECTRON MICROSCOPY LEVELS
}

\author{
F. J. CARRERAS ${ }^{1}$, J. J. LÓPEZ CABALLERO ${ }^{2}$ and D. PORCEL ${ }^{3}$ \\ Granada, Spain
}

\begin{abstract}
SUMMARY
A thin mucous layer covers the ciliary body and iris epithelium and becomes thicker in the anterior chamber where it covers the anterior surface of the iris, the chamber angle and corneal endothelial surface. It is especially thick at the chamber angle, where it adopts a meniscus shape with the concavity towards the anterior chamber. Cetylpiridinium chloride along with glutaraldehyde was used to precipitate this layer of mucous substance lining the anterior and posterior chambers of the human eye. The staining of the precipitate by cationic dyes suggests that glycosaminoglycans are main components. The specific characterisation with anti-hyaluronic acid monoclonal antibody labelled with colloidal gold reveals the presence of hyaluronic acid in the precipitate. Long unbranched hyaluronic acid molecules may form the skeleton of a gel able to trap and hold virtually any other macromolecule suspended in the aqueous humour. A possible role of such a gel in the regulation of aqueous humour outflow is discussed.
\end{abstract}

The localisation of the resistance to aqueous humour outflow remains controversial. A mathematical approach based on the accepted structural features of the exit pathways has shown that it is not possible to locate the cause of this resistance, despite the overwhelming physiological evidence tending to situate it in the trabecular meshwork. $^{1-4}$

The importance that glycosaminoglycans, and especially hyaluronic acid, may have in the resistance to outflow has been accepted since 1954 when Bàràny and Scotchbrook $^{5}$ showed a $50 \%$ reduction in the resistance to outflow after perfusion of the anterior chamber with

From: 'Department of Surgery (Ophthalmology), Faculty of Medicine, ${ }^{2}$ Department of Pathology, Faculty of Medicine, and ${ }^{3}$ Section of Electron Microscopy, Central Services, University of Granada, Granada, Spain.

Correspondence to: Dr F. J. Carreras, Departamento de Cirugía (Oftalmología), Facultad de Medicina, Avda. de Madrid, s/n 18071 Granada, Spain. hyaluronidase. They supported the claim by Meyer ${ }^{6}$ that glycosaminoglycans (GAGs) might have a regulating role in the control of aqueous flow. This line of thought was subsequently confirmed by several flow experiments in animal and enucleated human eyes. ${ }^{7-12}$

Clear morphological evidence that GAGs sensitive to hyaluronidase are present in the spaces of the trabecular meshwork was first provided by Zimmerman, ${ }^{13}$ and suggested in the indirect method used by Berggren and Vrabec $^{14}$ from a light microscopy study. These findings were subsequently disputed on the basis of observations both at the light microscopy $y^{15,16}$ and electron microscopy level. ${ }^{15}$ The use of cationic dyes in the study of GAGs by electron microscopy showed a positive staining only on the surface of the lining endothelium of the trabeculae and

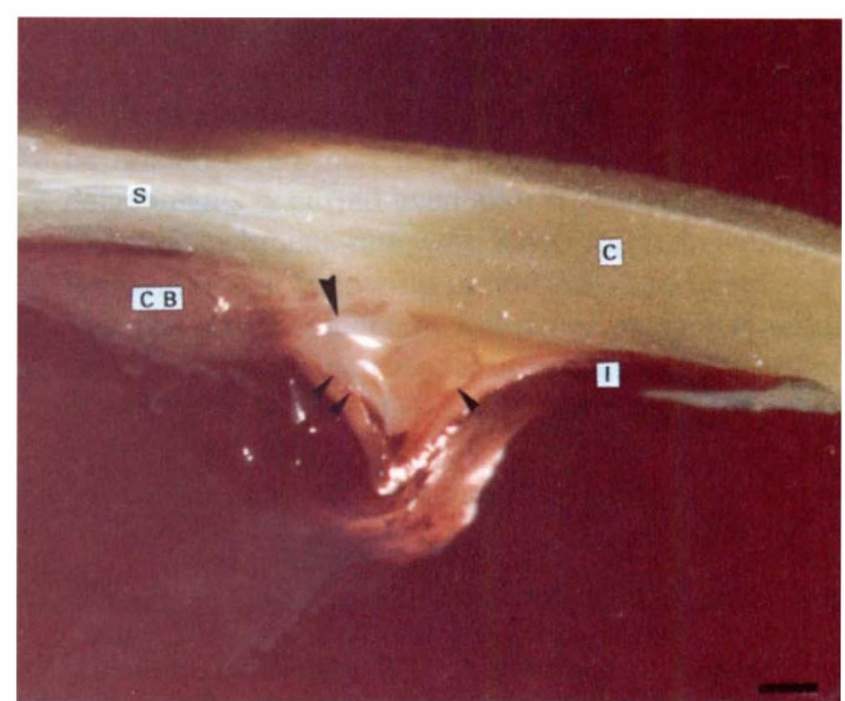

Fig. 1. Macroscopic appearance, after fixation using CPC, of a freshly enucleated eye. The picture shows a wedge-shaped piece containing cornea $(C)$, sclera $(S)$, iris (I) and ciliary body $(C B)$. The chamber angle is filled with a whitish substance (large arrowhead). This mucous substance also covers the anterior surface of the iris, from which is artefactually detached (small arrowheads). Scale bar represents $400 \mu \mathrm{m}$. 
a

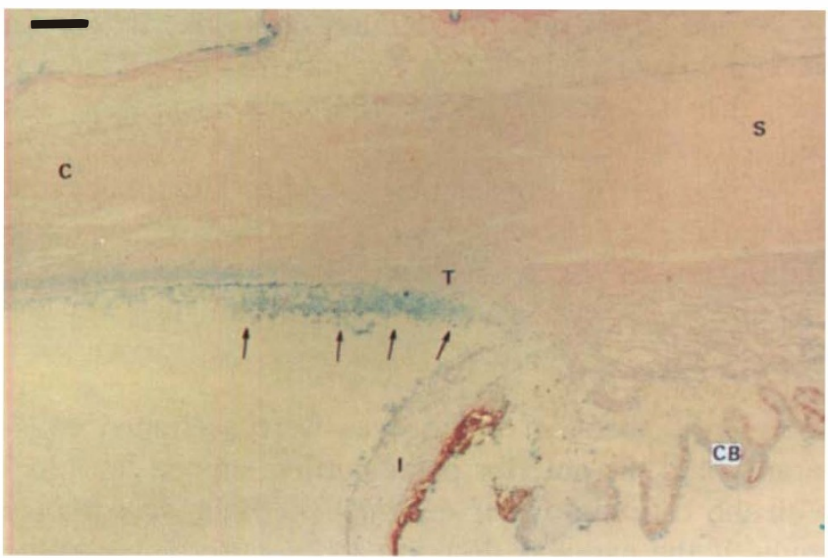

b

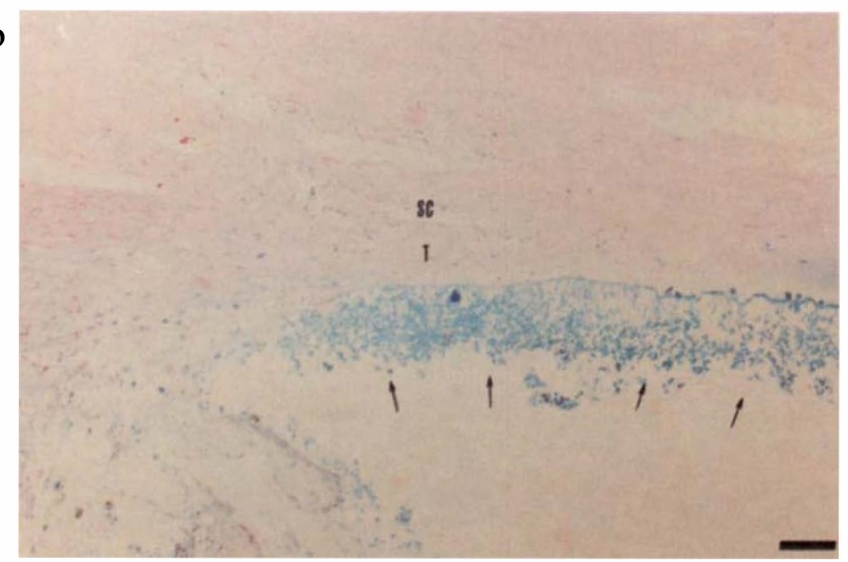

Fig. 2. (a) Light microscopy histological preparation showing the distribution of the mucous gel in the ocular chambers. GAGs have been stained with colloidal iron, turning prussian blue. The mucous gel is seen as a blue film covering the ciliary epithelium, the posterior iris epithelium, the surface of the iris stroma, the chamber angle (arrows) and the corneal endothelium. C, cornea; CB, ciliary body; I, iris; S, sclera; T, trabecular meshwork. Scale bar represents $250 \mu \mathrm{m}$. (b) A closer view showing the trabecular meshwork preceded by a thicker layer of mucosubstance (arrows). Schlemm's canal (SC) is open, the trabecular meshwork (T) is collapsed and some of the precipitate at the chamber angle is missing. Scale bar represents $100 \mu \mathrm{m}$. (Colloidal iron counterstained with nuclear red.)

on the internal wall of Schlemm's canal. ${ }^{17-23}$ Studies performed on various animal species, on the other hand, suggest that a layer of mucous substance is present covering the corneal endothelium ${ }^{22,24}$ or in the spaces of Fontana. $^{25,26}$
Discrepancies between results of different workers led us to suspect that differences in the method of fixation and staining of the tissue were critical when establishing the actual distribution of GAGs in the anterior chamber and outflow pathways.

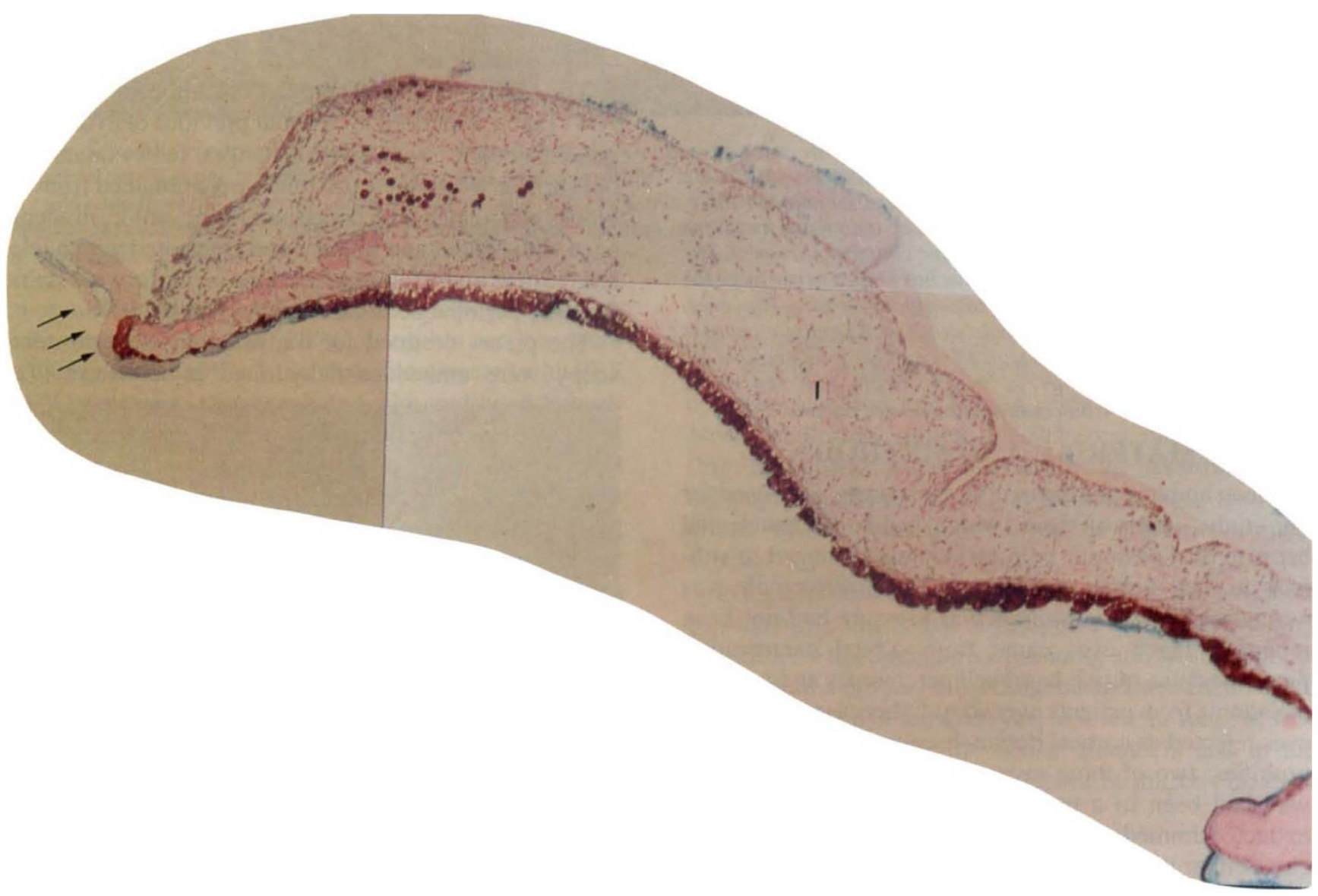

Fig. 3. Light microscopy histology of the iris (I). The mucous lining of the iris is very thin over the posterior epithelium and thicker at the pupillarymargin and surface of the iris stroma. The pupillary margin is surrounded by the layer of mucous gel like a well-fitted cuff. Part of the layer on the stromal surface of the iris has artefactually disappeared in some places. Scale bar represents $166 \mu$ m. (Copper and colloidal iron counterstained with nuclear red.) 


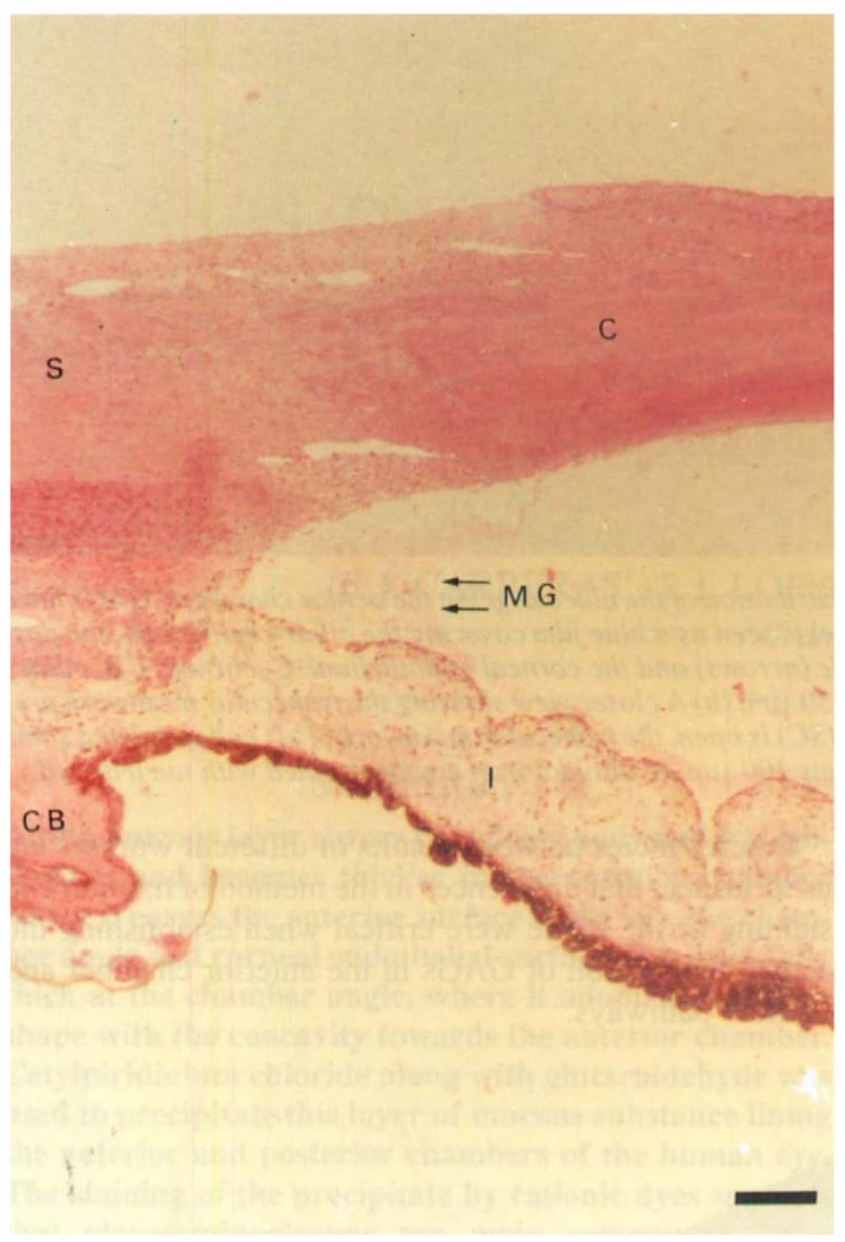

Fig. 4. Light microscopy histological preparation showing the layer of mucous gel (MG) reflecting over the chamber angle. The mucous gel layer adopts the shape of a concave meniscus with the concavity towards the anterior chamber (arrows). The presence of copperions in the fixative has helped to preserve the shape of the mucous gel lining the ocular chambers. However, copper competes with the cationic dyes for the anionic charges of the GAGs, and the gel has stained very faintly with cationised ferritin. Abbreviations as in Fig. 1. Scale bar represents $166 \mu \mathrm{m}$. (Colloidal iron counterstained with nuclear red.)

\section{MATERIAL AND METHODS}

Thirteen human eyes from different sources were used for our study. Three of them, which had to be enucleated because of a posterior pole melanoma, belonged to subjects in their thirties and forties. The anterior pole was normal and increased intraocular pressure had not been reported. Three eyes came from orbital exenteration pieces because of two basal cell carcinomas and a limbal melanoma from patients over sixty. Other cases came from eyes rejected as corneal donors because of minor corneal opacities: two of those eyes came from a young patient who had been in a traffic accident, and the eyes were extracted immediately after his death. In the remaining cases either the eyes had been stored at $4{ }^{\circ} \mathrm{C}$ for 24 hours or came from patients who had been in prolonged coma prior to death.

\section{Fixation Method}

A fixative containing $2 \%$ glutaraldehyde, $1 \%$ formalde- hyde and $0.5 \%$ cetylpiridinium chloride (CPC) in $0.05 \mathrm{~mol} / \mathrm{l}$ phosphate-buffered saline (PBS) at $\mathrm{pH} 7.4$ was used. ${ }^{27}$ In two of the cases $0.1 \%$ copper sulphate was added to the fixative.

Two eyes were split sagittally and fixed by immersion. The remaining eyes were fixed by perfusion of the fixative in the anterior chamber in the unopened eye, two cases at a pressure of $25 \mathrm{mmHg}$ and the rest at $6 \mathrm{mmHg}$.

\section{Staining Methods}

After fixation some of the eyes were sectioned equatorially and the anterior polar portion stained 'en bloc' with the cationic dye if destined for light microscopic study. If the eye was destined for electron microscopic study this step was omitted, and the specimen divided into wedges containing cornea, sclera, ciliary body and iris.

The staining was carried out by introducing the wedgeshaped pieces into a solution of colloidal iron, prepared according to Müller and Mowry, ${ }^{29}$ for 1 hour at room temperature, or into a solution of $250 \mu \mathrm{g} / \mathrm{ml}$ cationised ferritin (Sigma Laboratories) in PBS for 1 hour at $4{ }^{\circ} \mathrm{C} .{ }^{28}$

Some of the pieces stained with cationised ferritin were prepared for study under scanning electron microscopy. These pieces were postfixed at $-70{ }^{\circ} \mathrm{C}$ in a solution of $4 \%$ osmium tetroxide in absolute acetone, for 4 days. From this, they were gradually moved through different proportions of acetone/amyl acetate to $100 \%$ amyl acetate and critical-point-dried.

The pieces stained 'en bloc' were embedded in plastic JB4 (Taab Laboratories), without previous dehydration in graded alcohols, with several changes, for 24 hours without stirring. Sections, $2 \mu \mathrm{m}$ thick, were obtained from JB4 biocks and treated with potassium ferrocyanide, in accordance with Müller and Mowry, and contrasted with nuclear red. ${ }^{29}$ Some of the semithin sections in plastic were stained with the periodic acid-Schiff (PAS) method. ${ }^{29}$

The pieces destined for transmission electron microscopy were embedded-dehydrated in Lowicryl (Taab

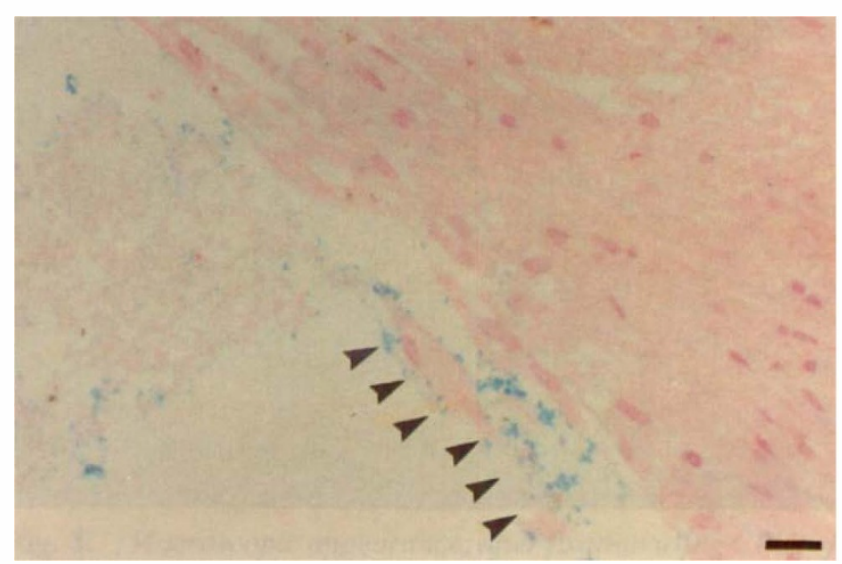

Fig. 5. Light microscopy histology of the trabecular meshwork. The mucous substance is only evident at the light microscope level in the first intertrabecular spaces of the uveal meshwork (arrowheads) and not in the corneoscleral meshwork. The trabecular meshwork is collapsed. Scale bar represents $166 \mu \mathrm{m}$. (Copper and colloidal iron counterstained with nuclear red.) 


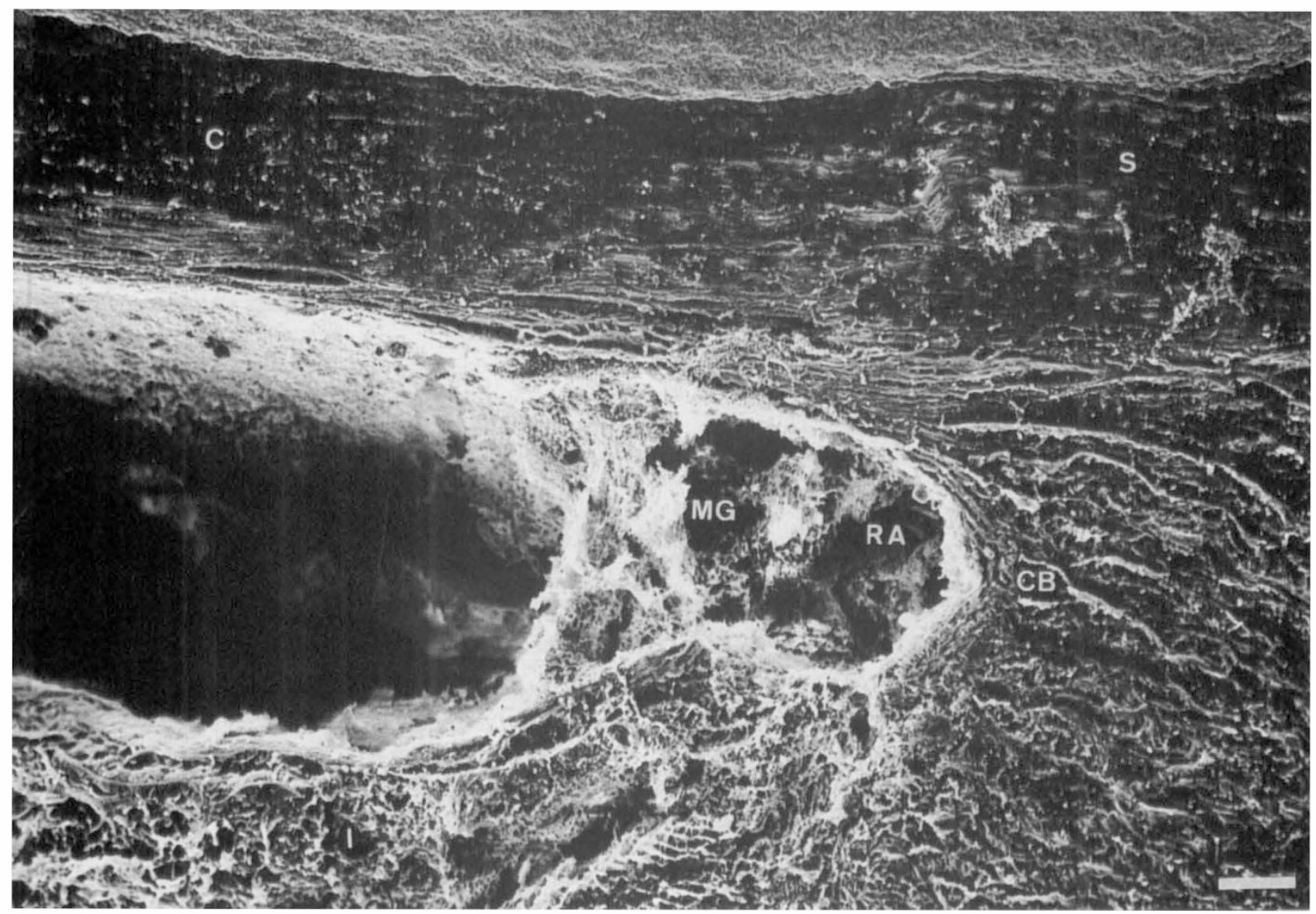

Fig. 6. Scanning electron micrograph of the chamber angle after precipitation with CPC and staining with cationised ferritin. The cornea is situated superiorly and the iris inferiorly. A thick layer $(M G)$ covers the anterior surface of the iris (I), fills the recessus angularis $(R A)$ and lines the posterior surface of the cornea $(C)$. The ferritin helps to provide support for the gel of GAGs preserving it as a three-dimensional network. The iris root is partially detached from the ciliary body $(C B)$ and the recessus angularis enlarged. Scale bar represents $19.2 \mu \mathrm{m}$.

Laboratories), with several changes before polymerisation under ultraviolet light at $-20^{\circ} \mathrm{C}$. Ultrathin sections were cut using a diamond blade. Sections were treated with PBS at $4{ }^{\circ} \mathrm{C}$ overnight, then incubated with undiluted hybridoma culture supernatant containing monoclonal IgM against hyaluronic acid (ND OG1, from Serotec) and secondarily incubated with a neat gold-labelled antimouse IgM antibody solution (Auroprobe EM GAMIgM G10, Janssen Laboratories). Once contrasted with uranyl acetate and lead citrate, sections were photographed with a transmission electron microscope (Zeiss EMI0C).

Sections not incubated with the monoclonal antibody and incubated with the gold-labelled probe were used as negative controls. As positive controls a portion of calf vitreous body was processed following the same steps employed with the anterior chamber mucous precipitate: fixation with CPC, dehydration-embedding in Lowicryl, and staining with the monoclonal antibody.

\section{RESULTS}

\section{Gross Examination}

Eleven eyes were fixed by intracameral perfusion and two by immersion. Five eyes of the first group and one of the second showed a whitish substance precipitating in the anterior chamber at the time of the fixation. The substance was clearly visible under the dissecting microscope, and could be seen as a fine film of white colour and mucous aspect uniformly lining the anterior chamber walls (Fig. 1). The mucosubstance covered the iris surface from the pupillary border up to the chamber angle and continued without interruption over the posterior corneal surface.

In the first cases studied, the mucous film was observed to vanish in some areas, or disappear completely, during the different manipulations of staining and washing of the tissues until a proper embedding method was established.

From the total of thirteen eyes, in seven the mucous substance precipitated in the ocular chambers and in the remaining six macroscopic evidence of mucous material was not found.

\section{Light Microscopy}

The sections of the tissues stained 'en bloc' with colloidal iron and further processed with potassium ferrocyanide showed a blue colour in those places where the iron had attached to polyanions. In the cases in which copper ions 
a

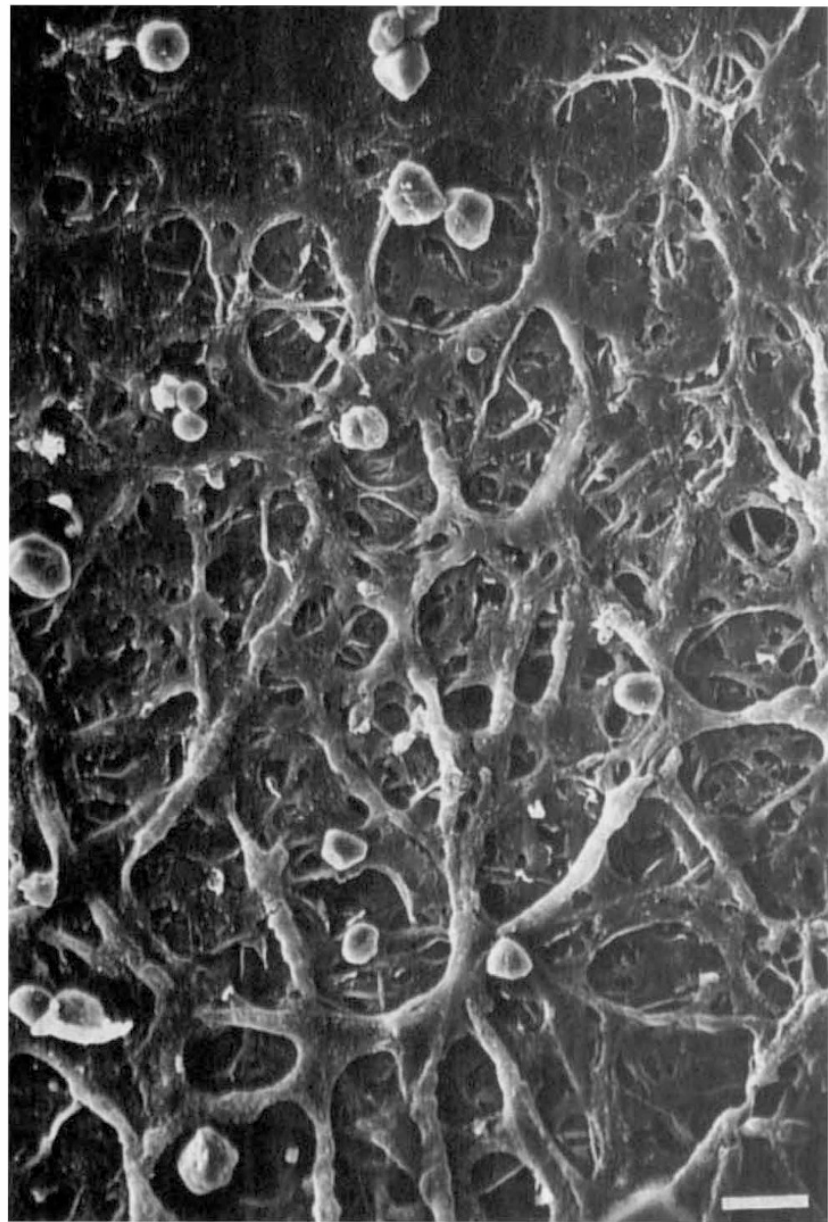

b

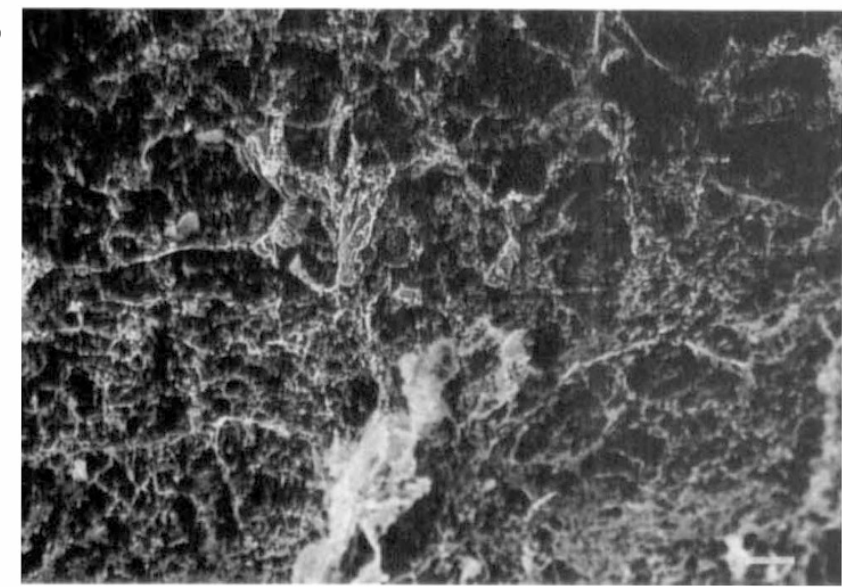

Fig. 7. Scanning electron micrographs. (a) Frontal view of the upper trabecular meshwork after conventional fixation. Scale bar represents $12.6 \mu \mathrm{m}$. (b) The same area after fuxation with CPC covered with a meshwork of mucosubstance reinforced with cationised ferritin. Scale bar represents $13.8 \mu \mathrm{m}$.

were present in the fixative the mucous material was better preserved but the blue staining was poorer. ${ }^{3}$

A thin blue film was apparent on the surface of the nonpigmented epithelial cells of the pars plicata of the ciliary body (Fig. 2). This film continuously covered the posterior epithelial surface of the iris up to the pupil. From the pupillary margin the film increased and extended as a thick mucous layer on the anterior surface of the iris
(Fig. 3). At the chamber angle the mucous layer passed over the drainage structures forming a concave meniscus. The angle recess of the anterior chamber was filled with this substance although some artefactual detachments were always present. The mucous layer became thinner and continued as a film over the endothelial surface of the cornea (Fig. 4). The intertrabecular spaces of the uveoscleral meshwork were occupied with the precipitate (arrowheads in Fig. 5). The intertrabecular spaces of the corneoscleral meshwork, normally collapsed when perfused at $6 \mathrm{mmHg}$ pressure, appeared empty (Fig. 5). This precipitate also gave a positive reaction with PAS.

The relationship between the mucous layer and the lens could not be observed in our preparations, because after fixing the eye the lens was systematically removed before staining.

\section{Scanning Electron Microscopy}

The study by scanning electron microscopy of two tissue pieces stained with cationised ferritin and postfixed by the freeze substitution method showed the angle of the anterior chamber fully occupied by a heavy-looking substance which extended in an irregular network over the anterior surface of the iris. The layer passed over the angle of the anterior chamber, forming a concave meniscus covering the trabecular meshwork, and continued as a thinner film spreading over the corneal endothelium (Figs. 6 and 7).

At a higher magnification the precipitate appeared as a three-dimensional mesh of interconnected clusters (Fig. 8).

\section{Transmission Electron Microscopy}

At low magnification the precipitate appears as clumps of amorphous substance interconnected by strands by variable widths. A neat and rather smooth surface is apparent facing the anterior chamber and, in some preparations, strands parallel to the cameral surface are seen in the mass of the substance occupying the angular recess (Fig. 9a).

At high magnification the same amorphous appearance remains. No specific structure or pattern is apparent in the strands and clumps when magnified 60000 times (Fig. 9b).

After incubation of the ultrathin sections of the anterior chamber precipitate with the monoclonal probe for hyaluronic acid, the labels gather on and around the clumps, and the substance-free areas appear void of gold (Fig. 10). The vitreous body appeared as irregular masses unevenly distributed, offering an image similar to the precipitate found in the anterior chamber. It showed also specific attachment of the monoclonal antibody. In no case did the negative controls show non-specific background.

The cells of the uveal and inner corneoscleral trabecular meshwork showed a disrupted cytoplasm with lysis of cellular organelles. Some of the nuclei were preserved, and others showed disintegration of the nuclear membrane and dispersion of the nuclear chromatin.

\section{DISCUSSION}

In 7 of the 13 eyes studied, a mucous substance was 


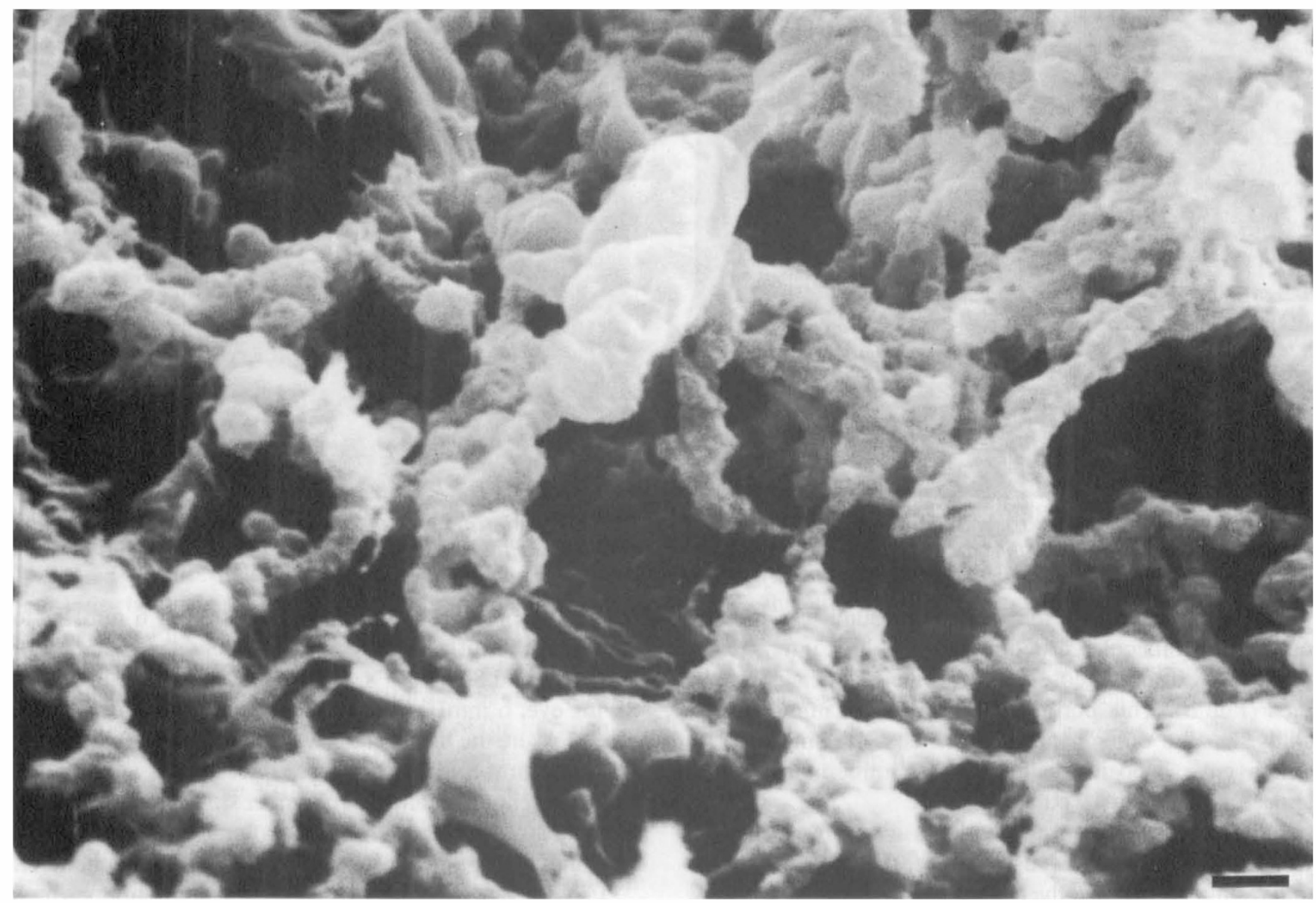

Fig. 8. Scanning electron micrograph showing a close-up view of the three-dimensional meshwork of mucosubstance, formed by interconnected amorphous clumps, covering the drainage pathways. Scale bar represents $0.95 \mu \mathrm{m}$. (Cationised ferritin.)

revealed covering the ciliary epithelium, the anterior and posterior surfaces of the iris, the posterior surface of the cornea and the chamber angle structures, being more abundant on the anterior surface of the iris and chamber angle.

In 1957 Zimmerman ${ }^{13}$ had already found a substance which rendered positive results with the metachromatic staining in the intertrabecular spaces of the trabecular meshwork. Its irregular distribution led him to believe that probably the whole trabecular meshwork was filled with the substance. He presented pictures of only one case, which could be interpreted as an indication of the random nature of the results obtained when conventional techniques of fixation were used. This interpretation seems to be borne out by the lack of later confirmation of the work of Zimmerman. a

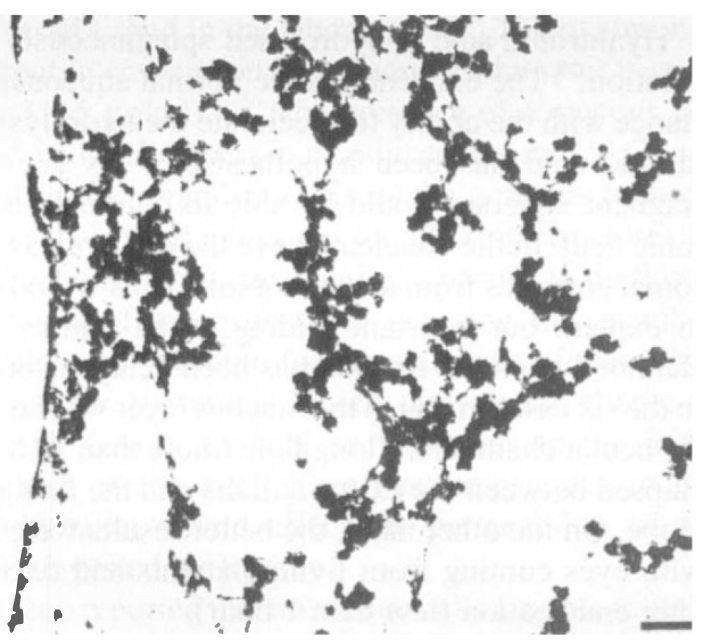

b

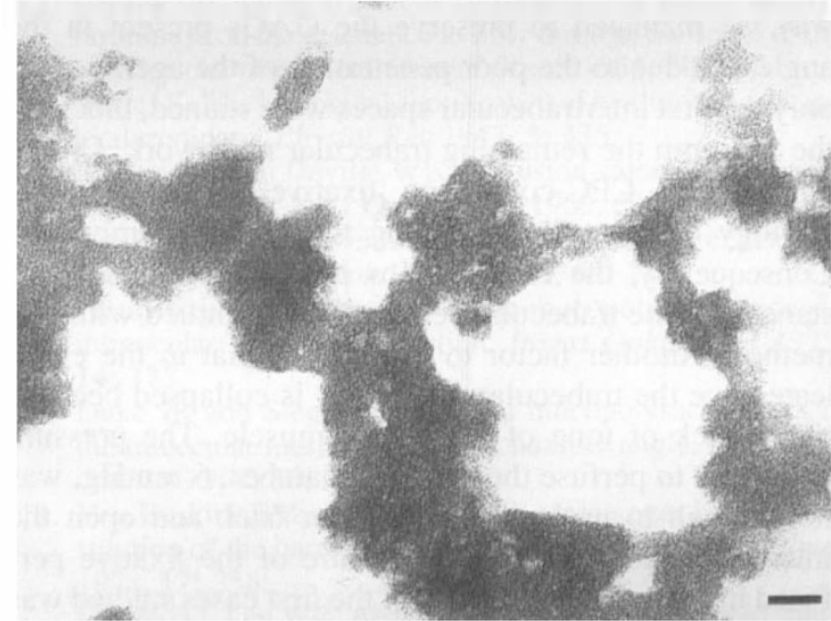

Fig. 9. Transmission electron micrograph of the mucous gel precipitated at the chamber angle by CPC containing fixative. (a) At low magnification the precipitate appears as small clumps interconnected by uneven strands. Scale bar represents $1.3 \mu m$. (b) At higher magnification, no fibrillar structure or banded pattern is apparent. Scale bar represents $0.1 \mu \mathrm{m}$. (Uranyl acetate, lead citrate.) 
a

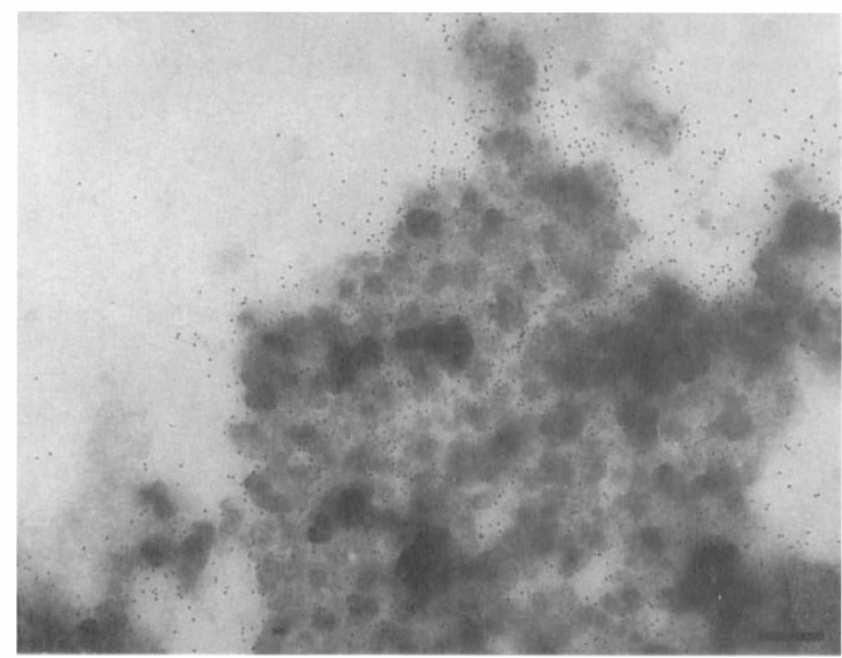

b

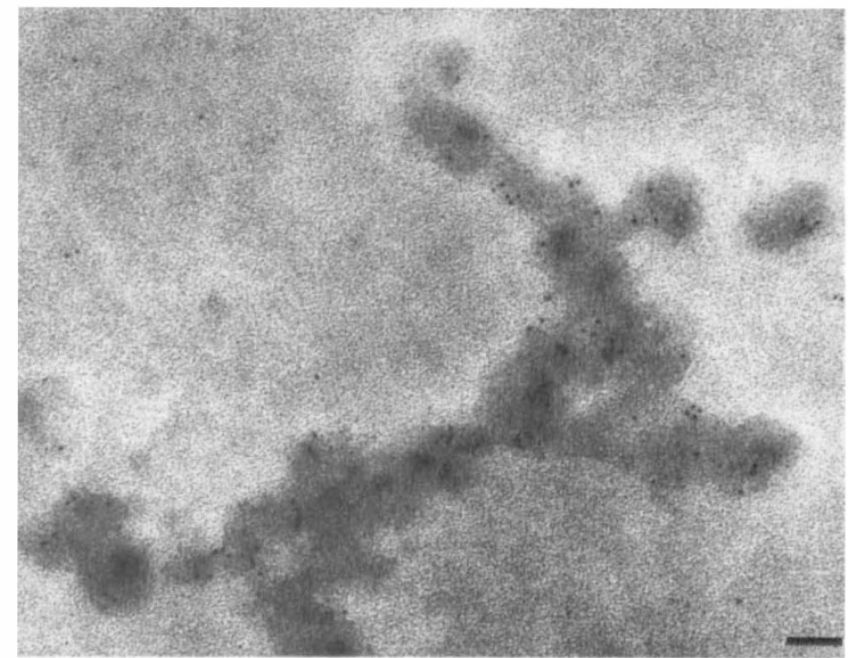

Fig. 10. Transmission electron micrograph of the mucous gel after characterisation of hyaluronic acid in the gel by means of a goldlabelled monoclonal anti-hyaluronic acid probe, in two steps, showing a specific distribution of the marks. Scale bar represents $0.22 \mu \mathrm{m}$ in (a) and $0.13 \mu \mathrm{m}$ in (b). (Monoclonal anti-hyaluronic acid IgM and gold-labelled anti-mouse IgM.)

In the present work, the substance found was mostly outside the trabecular structure. Only the first intertrabecular spaces of the uveoscleral meshwork appeared occupied (Fig. 5). This portion was empty in Zimmerman's study. On the other hand, the corneoscleral meshwork, which gave a positive staining in Zimmerman's case, appeared empty in all our cases.

Differences between our findings and those of other workers may justify a discussion of the methods employed to fix, stain and embed the tissues of the ocular chambers.

With regard to the pressure of perfusion of the anterior chamber, this discrepancy could be due to the fact that we fixed unopened eyes, cannulating the anterior chamber at a low pressure. The fixative had to cross through the pretrabecular mucous layer before gaining access to the intertrabecular spaces. The CPC could be retained by the pretrabecular gel and only CPC-free fixative permeated the trabecular meshwork. This interpretation is supported by the fact that only the cells of inner trabeculae showed the cytoplasm disrupted by the CPC, indicating that the external trabeculae had not been in contact with it. In this way we managed to preserve the GAGs present in the angle, but due to the poor penetration of the agents used, only the first intertrabecular spaces were stained, blocking the way into the remaining trabecular meshwork. On the other hand, CPC-containing fixatives alter the morphology of the cell, dissolving the lipidic membranes. Consequently, the relationships between the mucosubstance and the trabecular cell cannot be studied with this method. Another factor to consider is that in the enucleated eye the trabecular meshwork is collapsed because of the lack of tone of the ciliary muscle. The pressure employed to perfuse the anterior chamber, $6 \mathrm{mmHg}$, was not enough to push the scleral spur back and open the intertrabecular spaces. The pressure of the fixative perfused in the anterior chamber of the first cases studied was above $21 \mathrm{mmHg}$. With this high pressure the iris root is pushed backwards, the turbulence is noticeable and the mucous layer redissolved in some areas. In the last 11 cases the pressure was maintained around $6 \mathrm{mmHg}$, to simulate the difference in pressure between the anterior chamber and Schlemm's canal $(18 \mathrm{mmHg} / 12 \mathrm{mmHg}$ in a living eye; $6 \mathrm{mmHg} / 0 \mathrm{mmHg}$ in an enucleated eye). With this pressure the flow of fixative is much less turbulent and the structural relationships better preserved, although an enlargement of the anterior chamber still takes place probably due to the lack of choroidal volume in the enucleated eye and the absence of pressure in the posterior chamber.

$\mathrm{CPC}$ is a compound of quaternary ammonium which forms an insoluble complex with glycosaminoglycans and other polyanions. It is an effective agent in preventing the redistribution of hyaluronic acid which, because it is not present in the form of a proteoglycan, cannot be immobilised by the use of plain aldehydic fixatives. ${ }^{27,29}$

Part of this mucosubstance disappeared on occasions during the laboratory procedures, especially during dehydration. Using the embedding hydrosoluble medium as a dehydrating agent allows us to avoid the use of organic solvents, and that seems to be a critical step in the procedure.

Hyaluronic acid is hydrolysed spontaneously in water solution. ${ }^{30}$ The existence in the normal aqueous of a substance with the ability to accelerate the hydrolysis of hyaluronic acid has been hypothesised; ${ }^{31}$ its action in the stagnant aqueous would be able to eliminate the hyaluronic acid. In the enucleated eye the early release of lysosomal enzymes from the necrobiotic cells would also help to explain our constant finding of an 'empty' anterior chamber when the fixation has been delayed some hours. In the six cases in which the mucous layer was not found in the ocular chambers, a long time (more than 12 hours) had elapsed between the exitus lethalis and the fixation of the globe. On the other hand, the better results were obtained with eyes coming from living patients and fixed shortly after enucleation (less than 1 hour).

The affinity of the lining substance of the anterior chamber to cationic agents (colloidal iron and cationised ferritin) and its PAS positivity indicate its polyanionic 
nature. These staining procedures are normally used to detect glycosaminoglycans (also known as mucopolysaccharides). ${ }^{27-29}$

The evanescence of the precipitate, that faded away with a slight agitation of the fixative, and the fact that it was only found when CPC was used, and not with aldehydic fixatives alone, suggests that this is not a fibrin clot that can also be stained by the cationic dyes.

The ultrastructural approach showed an amorphous material forming aggregates of clumps and strands without a fibrillar or banded structure. This rules out fibrin, which is sometimes precipitated in post-mortem specimens and inflamed eyes.

Recently, a monoclonal antibody that specifically detects hyaluronic acid has been developed. ${ }^{32,33}$ In the eyes examined, the distribution of the gold-labelled monoclonal antibody against hyaluronic acid seems specific, indicating that hyaluronic acid is a main component of the mucous layer. On the other hand, it is worth noting the similarity between the material precipitated in the anterior chamber and the hyaluronic-acid-rich vitreous body processed with the same method, and used as a positive control for the characterisation of hyaluronic acid.

An important role in the maintenance of the intraocular pressure has been attributed to GAGs in the trabecular meshwork, and some efforts have been made to locate the main site of the resistance to aqueous outflow in the juxtacanalicular tissue, because this is the only portion of the trabecular meshwork in which the GAGs have so far been found in large enough quantities as components of the extracellular matrix. Some attempts have been made to attribute the flow resistance to the narrow channels through the extracellular substance of the juxtacanalicular tissue and through endothelial cells of the internal wall of the Schlemm's canal (giant vacuoles and transcellular channels), but physical analysis does not support this theory. ${ }^{3,4}$

Hyaluronic acid has the ability to form gels of randomly entangled molecules and is responsible for the main opposition to water flow in the matrix of connective tissue, as has been established in the laboratory. ${ }^{30,34}$ The hyaluronic acid present in suspension in the aqueous humour is considerable $(1-5 \mu \mathrm{g} / \mathrm{ml}) .^{35,36}$ The presence of a mucous gel lining the ocular chambers may indicate that the GAGs are deposited on the chamber walls as the aqueous humour flows over them, and mainly in the filtering structures of the chamber angle, thus originating a barrier to outflow.

Such a gel of mucosubstance covering the chamber angle could easily account for the main part of the resistance to outflow. Variations in size, density and charge of the layer of GAGs, as well as variations in the ionic strength and presence of other macromolecules in the aqueous humour, would affect the permeability of the layer, as suggested by Bàràny. ${ }^{31}$

It has been reported that trabecular cells are able to elaborate a wide range of GAGs. ${ }^{37,38}$ This is not surprising as these cells are responsible for the integrity of the trabeculae on which they rest. From this point of view they should play the role of fibroblasts renewing the fundamental matrix of the trabeculae. Also, by means of enzymic degradation, a variety of GAGs have been unmasked at the electron microscope level as forming part of the 'buffy coat' of the trabecular cells. ${ }^{39}$ Along with the intercellular matrix of the trabeculae the carbohydrates of the 'buffy coat' may be considered as the structural GAGs of the outflow pathways. On the other hand, the gel present in the chamber angle and filling the intertrabecular spaces may be considered as the osmotic GAGs, their principal role being the regulation of the aqueous flow.

The distribution of the gel suggests its origin in the posterior chamber, from where it could be carried in suspension in the aqueous humour and deposited in the chamber walls and drainage pathways. From Laurent's work we know that the hyaluronate present in the anterior chamber has a different molecular weight from the hyaluronate present in the vitreous body. ${ }^{40}$ The evidence that the ciliary epithelium is able to synthesise and secrete $\mathrm{GAGs}^{41,42}$ suggests that the gel's origin must be sought in the ciliary epithelium.

The authors wish to thank Professor W. R. Lee from the University of Glasgow for his invaluable assistance.

Key words: Aqueous humour outflow, Aqueous outflow resistance, Glycosaminoglycans, Trabecular meshwork.

\section{REFERENCES}

1. Tripathi RC: Mechanism of the aqueous outflow across the trabecular wall of Schlemm's canal. Exp Eye Res 1971, 11: 116.

2. Bill A and Svedbergh B: Scanning electron microscopic studies of the trabecular meshwork and the canal of Schlemm: an attempt to localise the main resistance to outflow of aqueous humour in man. Acta Ophthalmol (Copenh) 1972, 50: 295.

3. Eriksson A and Svedbergh B: Transcellular aqueous humour outflow: a theoretical and experimental study. Albrecht Von Graefes Arch Klin Exp Ophthalmol 1980, 212: 53.

4. Moseley H, Grierson I, Lee WR: Mathematical modelling of aqueous humour outflow from the eye through the pores in the lining endothelium of Schlemm's canal. Clin Phys Physiol Meas 1983, 4: 47.

5. Bàràny $\mathrm{EH}$ and Scotchbrook $\mathrm{S}$ : Influence of testicular hyaluronidase on the resistance to flow through the angle of the anterior chamber. Acta Physiol Scand 1954, 30: 240.

6. Meyer K: The biological significance of hyaluronic acid and hyaluronidase. Physiol Rev 1947, 3: 335.

7. Melton CE and Deville WB: Perfusion studies on the eyes of four species. Am J Ophthalmol 1960, 50: 302.

8. Grant WM: Experimental aqueous perfusion in enucleated human eyes. Arch Ophthalmol 1963, 69: 783.

9. François J: The importance of the mucopolysaccharides in intraocular pressure regulation. Invest Ophthalmol 1975, 14: 173 .

10. Duke JR and Siègelman S: Acid mucopolysaccharides in the trabecular meshwork of the chamber angle. Arch Ophthalmol 1961, 66: 399.

11. Van Buskirk EM and Brett J: The canine eye: in vitro dissolution of the barriers to aqueous flow. Invest Ophthalmol 1978, 17: 258.

12. Grierson I, Lee WR, Abraham S: A light microscopic study of the effects of testicular hyaluronidase on the outflow system of the baboon (Papio cynocephalus). Invest Ophthalmol 1979, 18: 356. 
13. Zimmerman LE: Demonstration of hyaluronidase sensitive acid mucopolysaccharides in trabecula and iris in routine paraffin sections of adult human eyes. Am J Ophthalmol 1957, 44: 1.

14. Berggren L and Vrabec F: Demonstration of a coating substance in the trabecularmeshwork of the eye. Am J Ophthalmol 1957, 44: 200.

15. Segawa K: Localisation of acid mucopolysaccharides in the human trabecular meshwork. Jpn J Clin Ophthalmol 1970, 24: 363.

16. Armaly MF and Wang Y: Demonstration of acid mucopolysaccharides in the trabecular meshwork of the rhesus monkey. Invest Ophthalmol 1975, 14: 507.

17. Grierson I and Lee WR: Acid mucopolysaccharides in the outflow apparatus. Exp Eye Res 1975, 21: 417.

18. Grierson I, Lee WR, Abraham S: The appearance of the outflow apparatus of the eye after staining with ruthenium red. Acta Ophthalmol 1977, 55: 827.

19. Harnish JP: Electron microscopical delineation of acid mucopolysaccharides in the trabecular meshwork. Klin Monastsbl Augenheilkd 1976, 169: 90.

20. Mizokami K: Demonstration of masked acidic glycosaminoglycans in the normal trabecular meshwork. Jpn J Ophthalmol 1977, 21: 57.

21. Bàràny $\mathrm{E}$, Berggren $\mathrm{L}$, Vrabec $\mathrm{F}$ : The mucinous layer covering the corneal endothelium in the owl Strix aluco. $\mathrm{Br}$ J Ophthalmol 1957, 41: 25.

22. Wolf J: Inner surface of regions in the anterior chamber taking part in the regulation of the intraocular tension including the demonstration of the covering viscous substance. Doc Ophthalmol 1968, 25: 113.

23. Kneper PA, Farbman AJ, Telser AG: Aqueous outflow pathway glycosaminoglycans. Exp Eye Res 1981, 32: 265.

24. Carrington SD, Alexander RA, Grocott P, Grierson I: The viscous layer overlying the corneal posterior epithelium of the domestic cat. J Anat 1987, 153: 77.

25. Morrison JC and Van Buskirk EN: The canine eye: pectinate ligaments and aqueous outflow resistance. Invest Ophthalmol Vis Sci 1982, 23: 726.

26. Richardson TM: Distribution of glycosaminoglycans in the aqueous outflow system of the cat. Invest Ophthalmol Vis Sci 1982, 22: 319

27. Scott JE: The precipitation of polyanions by long-chain aliphatic ammonium salts. VI. The affinity of substituted ammonium cations for the anionic groups of some biological polymers. Biochem J 1965, 84: 270.

28. Danon D, Goldstein L, Marikousky Y, Skutelsky L: Use of cationised ferritin as a label of negative charges on cell surfaces. J Ultrastruct Res 1972, 38: 500.

29. Kupchella CE, Matsuoka LY, Bryan B, Wortsman J, Dietrich JG: Histochemical evaluation of glycosaminoglycan deposition in the skin. J Histochem Cytochem 1984, 32: 1121.

30. Laurent TC: Structure of hyaluronic acid. In: Balazs EA, ed. Chemistry and Molecular Biology of the Intercellular Matrix. Vol. 2. London: Academic Press, 1970.

31. Bàràny EH: Physiological and pharmacological factors influencing the resistance to aqueous outflow. In: Newell FW, ed. Glaucoma. Transactions of the First Josiah Macy Foundation, 1956:123.

32. Sunderland CA, Redman CWG, Stirrat GM: Monoclonal antibodies to human syncytiotrophoblast. Immunology 1981, 43: 541-6.

33. Sunderland GA, Bulmer JN, Lunscombe M, Redman CWG, Stirat GM: Immunohistological and biochemical evidence for a role for hyaluronic acid in the growth and development of the placenta. J Reprod Immnol 1985, 8: 197-212.

34. Ogston AG, Sherman TF: Effect of hyaluronic acid upon diffusion of solutes and flow of solvent. J Physiol 1961, 156: 67.

35. Laurent UBG: Hyaluronate in aqueous humour. Exp Eye Res 1981, 33: 147.

36. Österlin S: Macromolecular composition of the vitreous in the afakic owl monkey eye. Exp Eye Res 1978, 26: 77.

37. Schachstchabel DO, Rohen JW, Wever J, Sames K: Synthesis and composition of glycosaminoglycans by cultured human trabecular meshwork cells. Albrecht von Graefes Arch Klin Exp Ophthalmol 1982, 218: 113.

38. Polansky J, Gospadorowicz D, Weinreb R, Alvarado J: Human trabecular meshwork cell culture and glycosaminoglycans synthesis. Invest Ophthalmol Vis Sci 1978, 17: 207.

39. Mizokami K: Demonstration of masked acidic glycosaminoglycans in the normal trabecular meshwork. Jpn J Ophthalmol 1977, 21: 57.

40. Laurent UBG and Frazer JRE: Turnover of hyaluronate in aqueous humour and vitreous body of the rabbit. Exp Eye Res 1983, 36: 493.

41. Fine BS and Zimmerman LE: Light and electron microscopic observations on the ciliary epithelium in man and rhesus monkey. Invest Ophthalmol 1963, 2: 105.

42. Raviola G: Evidence for a secretory process, distant from that of the aqueous humour, in the ciliary epithelium of Macaca mulatta. Trans Ophthalmol Soc UK 1986, 105: 140. 